\title{
A Literature Review: Detection of COVID-19 in Computed Tomography Images Using Deep Learning
}

\author{
Júlio V M Marques ${ }^{1}$, Rodrigo M S Veras ${ }^{1,2}$, Romuere R V Silva ${ }^{1,3}$ \\ ${ }^{1}$ Programa de Pós-Graduação em Ciência da Computação \\ Universidade Federal do Piauí (UFPI) - Teresina - PI - Brasil \\ ${ }^{2}$ Departamento de Computação - UFPI - Teresina - PI - Brasil \\ ${ }^{3}$ Departamento de Sistemas de Informação - UFPI - Picos - PI - Brasil \\ \{juliomonteiro, rveras, romuere\}@ufpi.edu.br
}

\begin{abstract}
Through the development of the COVID-19 disease, various diagnosis methods have been studied. One of them is the computed tomography (CT), which has the best level of detail among medical image exams. The CT generates a repeatable and tiring workload, in addition to needing a team that is familiar with the findings that indicate pneumonia caused by COVID-19. To reduce this manual work and collaborate with these teams, several studies have been carried out using deep learning techniques. In this way, this study presents a review of the literature regarding the detection of COVID-19 in CT that uses deep learning to collaborate with a theoretical basis for future works.
\end{abstract}

\section{Introdução}

A COVID-19 é uma doença causada pelo vírus SARS-COV-2, e até agosto de 2021, levou a óbito um total de 4,4 milhões de pessoas e possui um total de casos confirmados de 211 milhões [Organization 2020]. Os sintomas da doença variam de casos leves e recuperação rápida, até estados graves, causando pneumonia e falta de ar, e alguns casos podem levar a doenças críticas, incluindo insuficiência respiratória [Mahase 2020].

O diagnóstico em estágio inicial é imprescindível e o meio mais utilizado é o exame da reação em cadeia da polimerase com transcrição reversa em tempo real (RTCPR) [Wang et al. 2020a]. Porém, a disponibilidade desses testes são limitados, com isso outros métodos de diagnóstico tem sido amplamente estudados, como radiografia e tomografia computadorizada (TC). Dentre esses, a radiografia é a mais comumente disponível. Entretanto, para a COVID-19, a radiografia apresenta um desempenho limitado, devido a sua baixa sensibilidade e especificidade [Farias et al. 2020]. Com isso, a TC é vista como um meio mais eficaz e confiável devido ao seu nível de detalhes, porém o processo manual usado para analisar as imagens de TC do tórax torna esse trabalho cansativo, além de necessitar de uma equipe familiarizada com os achados nas imagens que são sugestivos da pneumonia viral compatível com COVID-19.

O presente trabalho apresenta uma revisão bibliográfica das pesquisas que se utilizam de técnicas com aprendizado profundo para a classificação desses achados nas imagens de TC. Tais técnicas tem por objetivo diminuir o processo manual de análise dessas imagens e o trabalho cansativo, além de reduzir a necessidade de uma equipe familiarizada com os achados nas imagens. Os principais objetivos desse trabalho são: 1) identificar as principais bases de imagens públicas; 2) identificar as arquiteturas de aprendizado 
profundo que obtiveram os melhores resultados; 3) realizar um levantamento das técnicas de aumento de dados, ajuste fino, segmentação de imagens e pré-processamento das imagens. Todas essas informações contribuem para um embasamento teórico que pode vir a auxiliar no desenvolvimento de sistemas computacionais e aplicações que contribuem para o auxílio do diagnóstico da COVID-19 em imagens de TC.

\section{Fundamentação Teórica}

\subsection{Tomografia Computadorizada}

A TC consiste em uma fonte de raios-X que é acionada em movimentos circulares, emitindo feixes de raios- $\mathrm{X}$ em forma de leque. No lado oposto dessa fonte existem detectores que transformam a radiação em sinais elétricos e posteriormente em imagens digitais [Júnior and Yamashita 2001]. Em um estudo de comparação da técnica molecular dos autores [Farias et al. 2020], a TC de tórax mostra uma sensibilidade de $97 \%$ na detecção da COVID-19. Isso demonstra que a TC de tórax tem um desempenho próximo ao RTPCR para a COVID-19, provando ser um meio eficaz e confiável.

\subsection{Aprendizado Profundo}

O Aprendizado Profundo é uma subárea da inteligência artificial, que oferece meios para analisar áudios, textos, conteúdos visuais como vídeos e imagens [Ponti and da Costa 2017]. Para o exame da TC de tórax, a principal técnica de aprendizado profundo são as Redes Neurais Convolucionais (CNN), essas redes possuem uma arquitetura profunda e hierárquica, capaz de extrair características dos dados de entrada e representá-las como informações, desde as representações mais simples às mais complexas e classificá-las. As CNNs são compostas por três tipos de camadas: convolução, pooling e totalmente conectadas. Nas camadas de convoluções temos a extração de características. Após isso, as características passam para as camadas de pooling que são responsáveis por uma simplificação dessas informações, reduzindo a dimensão dos dados e acelerando o processamento para a próxima camada. Por fim, as camadas totalmente conectadas interpretam as características que foram geradas nas camadas anteriores para classificá-las. Na Figura 1 temos uma representação simples da estrutura de uma CNN.

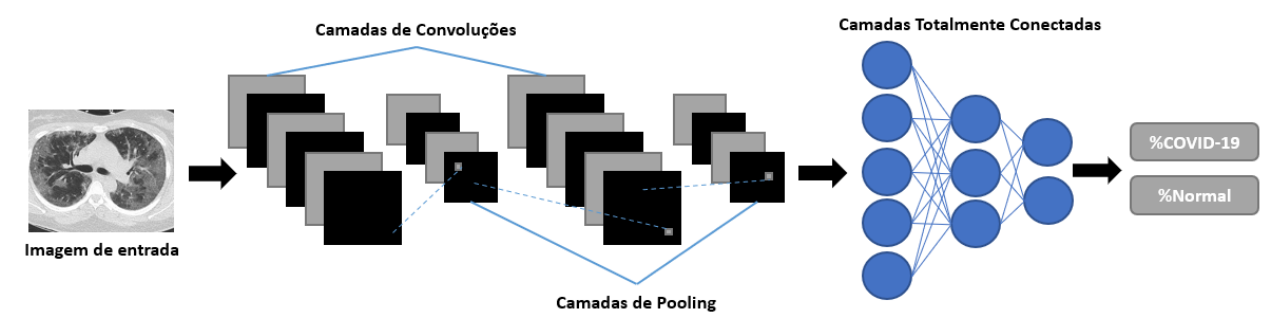

Figura 1. Representação da estrutura básica de uma CNN.

\section{Metodologia}

Para a realização da revisão de literatura, foram utilizada as etapas propostas por Kitchenham [Kitchenham 2004], são elas: planejamento, condução, e extração dos dados. Para o planejamento, partimos das seguintes questões: "Quais as bases de imagens médicas os 
autores estão utilizando?", "Quais as principais técnicas de aprendizado profundo estão sendo empregadas?", "Quais das CNNs utilizadas tem o melhor desempenho?". Definimos os seguintes critérios para Inclusão (I) e Exclusão (E) de trabalhos: (I) Se o artigo emprega técnicas de visão computacional ou processamento digital em imagens de TC relacionadas à COVID-19; (I) Se o artigo realiza uma detecção da pneumonia causada pela COVID-19, utilizando aprendizado profundo em imagens de TC; (I) Estudos a partir de 2020; (E) Se possui idioma diferente do inglês; (E) Se não possui uma metodologia para solução do problema. Na etapa de condução, submetemos a string de busca ("deep learning" OR "convolutional neural network") AND (COVID-19 OR SARS-COV-2 OR coronavirus) AND (CT OR computed tomography) nas bases IEEE Xplorer, Scielo, Science Direct e PubMed, resultando em 189 artigos retornados e, por fim, um total de 30 artigos foram selecionados na última etapa. Na Figura 2 apresentamos todas as etapas e os filtros utilizados durante a etapa de condução. As informações extraídas dos trabalhos selecionados são apresentados na Seção 4.

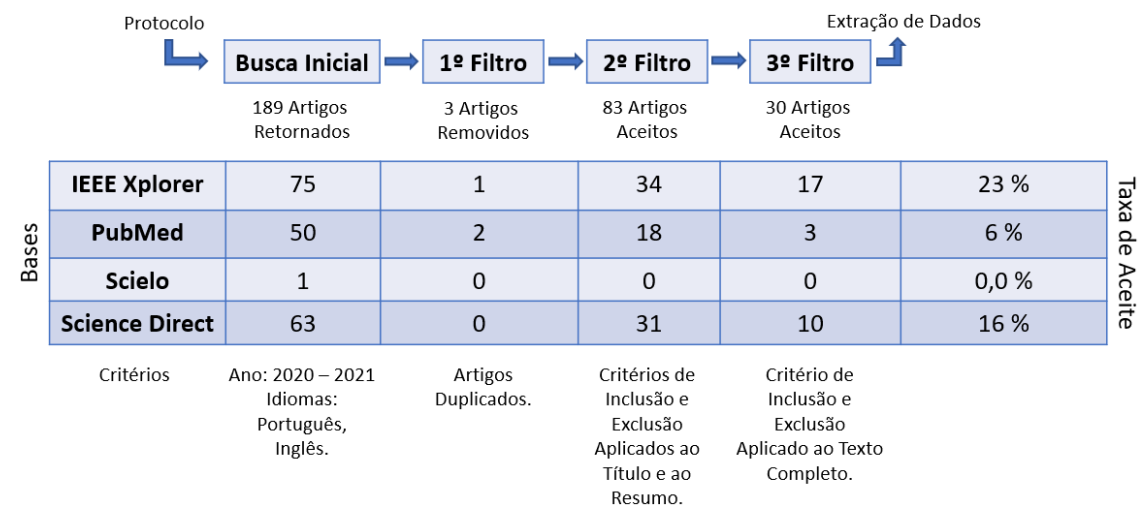

Figura 2. Etapas de filtragem da revisão bibliográfica.

\section{Resultados}

Técnicas de aprendizado profundo podem oferecer meios viáveis para solucionar problemas em um ambiente real. Dessa forma, apresentamos como resultados os principais trabalhos encontrados utilizando tais técnicas em imagens de TC. Além disso, apresentamos também as principais bases de imagens disponíveis publicamente.

\subsection{Detecção da COVID-19: Tomografias Computadorizadas}

Detalhamos a seguir os principais trabalhos que encontramos utilizando técnicas de aprendizado profundo em imagens de TC.

Vários autores implementaram modelos próprios baseados em outros já existentes na literatura, entre eles temos, os autores [Lai et al. 2020], que propõem uma abordagem de detecção e classificação das lesões. O método inclui segmentação, detecção de lesões e classificação de doenças. Em [Sari et al. 2020], os autores trazem uma proposta de um novo modelo cujo métricas superam modelos mais conhecidos. Os autores [Zhang et al. 2020], apresenta uma proposta de modelo denominado de 7L-CNN-CD unido com um método para o pré-processamento das imagens. Na abordagem de [Wang et al. 2020b], é proposto um modelo para prever a probabilidade de infecção da COVID-19 e encontrar lesões na TC de tórax. No trabalho proposto 
em [Castiglione et al. 2021], é apresentada uma abordagem de pré-processamento de imagens e trazem também um modelo otimizado denominado de ADECO-CNN. Já o trabalho de [Islam and Matin 2020] apresenta o modelo LeNet-5, juntamente com a técnica de aumento de dados e um pré-processamento das imagens. Em [Gunraj et al. 2020], trazem um novo modelo (COVIDNet-CT). Esse modelo foi treinado previamente no conjunto de dados da ImageNet, depois usando a transferência de aprendizagem o modelo foi usado no conjunto de imagens de TC. No trabalho dos autores [Rahimzadeh et al. 2021], é proposta uma nova rede baseada na ResNet50V2 em pirâmide de atributos. No estudo de [Hu et al. 2021], é empregado um novo modelo com perda auxiliar auto-adaptativa (DSN-SAAL) para a classificação da COVID-19 em imagens de TC. Em [Di et al. 2021], é apresentado um método de Hypergraph Learning com Vértice de Incerteza (UVHL) para identificação de COVID-19. Em [Zhou et al. 2021], é apresentado um modelo de aprendizado profundo para detecção de COVID-19 em imagens de TC, utilizando aprendizado por transferência são treinados três modelos de $\mathrm{CNN}$, foi obtido com isso o modelo EDL-COVID. Onde o desempenho geral mostrou que o EDL-COVID foi melhor do que um único classificador.

Seguindo os autores citados acima, temos aqueles que fizeram uso de modelos prétreinados com algumas outras técnicas, como os autores [Dan-Sebastian et al. 2020], que trazem um novo procedimento de treinamento, denominado Adversarial Graph Learning (AGL), sendo avaliado em duas CNNs. O trabalho feito pelos autores [Abdar et al. 2020], apresenta uma metodologia para o pré-processamento das imagens de TC e a arquitetura VGG16 é utilizada para receber um ajuste fino. Já os autores em [Wang et al. 2020c], apresentam um reprojeto da COVID-Net, desenvolvida originalmente para imagens de raio-x, essa abordagem foi capaz de superar o COVID-Net. Já em [Berrimi et al. 2021], é feito o uso de modelos com aprendizagem por transferência, aumento de dados e ajuste fino. No estudo feito pelos autores [Dutta et al. 2021], foi utilizada a Inception V3, com transferência de aprendizagem, aumento de dados, ajuste fino e ajuste de hiperparâmetros. Já os autores [Carvalho et al. 2020], trazem uma versão simplificada da rede LeNet-5 para extrair as características das imagens unido com o XGBoost para a classificação. No estudo proposto por [Kamel et al. 2021], é apresentada uma metodologia para o pré-processamento das imagens juntamente do modelo VGG-19 com a transferência de aprendizagem e ajuste fino para classificação das imagens. Já em [Cruz 2021] e [Mishra et al. 2021], são empregadas técnicas de transferência de aprendizagem com o aumento de dados e uma CNN combinada. No trabalho de [Jia et al. 2021], é empregada uma arquitetura ResNet modificada para classificação de imagens de TC. O trabalho realizado por [Turkoglu 2021], apresenta um método em Multiple Kernels-ELM (MKs-ELMDNN), para a detecção de COVID-19 por meio de imagens de TC de tórax utilizando a rede DenseNet201. Já na pesquisa dos autores [Li et al. 2021], foi proposto um método (CheXNet) baseado em transferência de aprendizagem que visa identificar COVID-19 em imagens de TC de tórax. No trabalho de [Arora et al. 2021], é feito o uso da Super Resolução (SR) no pré-processamento das imagens com técnicas de aumento de dados e a transferência de aprendizagem. Foi possível observa que o uso da SR melhorou os resultados de modelos como a MobileNet.

Por fim temos os autores que fizeram uma revisão dos modelos mais difundidos da literatura, como os autores [Seum et al. 2020], que apresentam uma avaliação de diferentes CNN pré-treinadas, com destaque para ResNet18 e DenseNet121 entre os mo- 
delos com e sem segmentação, respectivamente. Em [Cai et al. 2020] é realizado um estudo de três tipos de ResNet, onde a ResNet-18 obteve os melhores resultados. Os autores em [Yener and Oktay 2020] trazem um estudo sobre três modelos populares de CNN usando aprendizagem por transferência, onde a VGG16 e VGG19 obtiveram resultados semelhantes e melhores que a Xception. Os autores [Serte and Serener 2020] tiveram como objetivo distinguir COVID-19 de outras infecções. Dentre as CNNs utilizadas, a ResNet-50 se destacou, para todos casos ela obteve os melhores resultados. Os autores em [Ardakani et al. 2020] e [Gifani et al. 2021] apresentam um estudo com dez e quinze CNNs respectivamente, onde as Resnet-101, Xception, EfficientNet e InceptionV3 se destacaram.

\subsection{Bases de Imagens}

A maior dificuldade para desenvolver pesquisas com CNN no contexto do novo coronavírus em imagens de TC é a falta de dados. Devido a esse problema, várias bases de imagens foram criadas. Dessa forma, apresentamos aqui as duas maiores bases de imagens disponíveis publicamente até o momento. A COVIDCTset [Rahimzadeh et al. 2021], e a COVIDx-CT-2 [Gunraj et al. 2020]. O dataset COVIDx CT-2, compreende 194.922 cortes de TC de 3.745 mil pacientes, sendo este o maior conjunto de imagens disponível no momento. Ele é dividido em três classes, 60.083 imagens normais, 40.291 imagens de pneumonia e 94.548 imagens de COVID-19 e está disponível no Kaggle ${ }^{1}$. Já o dataset COVID-CTset contém 15.589 imagens de COVID-19 (95 pacientes) e 48.260 imagens normais (282 pacientes) e está disponível no GitHub ${ }^{2}$.

\subsection{Discussões}

Na Tabela 1 apresentamos os resultados obtidos nos trabalhos pesquisados e as principais técnicas empregadas. Na tabela temos indicações de quais trabalhos aplicaram préprocessamento de imagens (PPI), aprendizado por transferência (TA), aumento de dados (DA) e algoritmos de segmentação (S). Dentre os trabalhos apresentados, 76,66\% utilizam algoritmos de pré-processamento, 53,33\% fazem uso do aprendizado por transferência, e $30 \%$ utilizam a técnica de aumento de dados. Apenas 6,66\% dos trabalhos realizam segmentação. Podemos destacar o trabalho de [Arora et al. 2021], com a maior acurácia (100\%), precisão (100\%) e F1-score (100\%). Outro trabalho que pode ser destacado é o dos autores [Hu et al. 2021], onde foi possível atingir a maior AUC (99,95\%), e os autores [Ardakani et al. 2020], conseguiram atingir a maior sensibilidade (100\%).

\section{Conclusão}

Nesse trabalho, realizamos um levantamento de pesquisas que fazem uso do aprendizado profundo para detectar a pneumonia causada pelo novo coronavírus. Concluiu-se que independente da modalidade e abordagens dos autores nas imagens utilizadas, as técnicas obtiveram resultados significativos em diferentes métricas e quando utilizas com algum tipo de pré-processamento onde se obtém as regiões de interesses das imagens as CNNs ganham um poder maior de classificação. Além disso, foi possível identificar dois conjunto de dados que resolvem o desafio da quantidade de dados inerentes ao treinamento de CNNs. Como trabalhos futuros, pretendemos investigar o desempenho dos modelos

\footnotetext{
${ }^{1}$ https://www.kaggle.com/hgunraj/covidxct

${ }^{2}$ https://github.com/mr7495/COVID-CTset
} 
Tabela 1. Resumo dos trabalhos, técnicas empregadas e seus resultados.

\begin{tabular}{|c|c|c|c|c|c|c|c|}
\hline Métodos & Técnicas & $\operatorname{Acc}(\%)$ & Sens(\%) & $\operatorname{Espec}(\%)$ & Prec(\%) & F1(\%) & $\operatorname{AUC}(\%)$ \\
\hline Lai et al. 2020 (S, DA) & Modelo Próprio & 86,1 & 75,7 & 95,2 & - & - & 91,0 \\
\hline Sari et al. 2020 (PPI) & Modelo Próprio & 97,5 & - & - & 98,0 & 98,0 & - \\
\hline Seum et al. 2020 (PPI, S, TA) & U-Net + ResNet18 & 89,9 & 80,4 & 99,5 & 99,5 & 88,9 & - \\
\hline Zhang et al. 2020 (PPI, DA) & Modelo Próprio & 94,0 & 94,4 & 93,6 & - & 94 & - \\
\hline Wang et al. 2020b (S, PPI, DA) & U-Net + Modelo Próprio & 90,1 & - & - & - & - & 95,9 \\
\hline Dan-Sebastian et al. 2020 (PPI, TA) & Xception & 78,4 & - & - & - & - & 84.69 \\
\hline Abdar et al. 2020 (PPI, TA) & VGG16 & 90,1 & - & 90,5 & 91,5 & 90,6 & - \\
\hline Cai et al. 2020 (PPI) & ResNet18 & 94,3 & 91,4 & 97,3 & 97,1 & 94,2 & 98,5 \\
\hline Wang et al. 2020c & COVID-Net & 90,8 & - & - & 95,7 & 90,8 & 96,2 \\
\hline Castiglione et al. 2021 (PPI) & Modelo Próprio & 99,9 & 99,9 & 99,9 & 99,9 & - & - \\
\hline Berrimi et al. 2021 (PPI, DA, TA) & DenseNet & 95,9 & - & - & - & - & - \\
\hline Dutta et al. 2021 (TA, DA) & Inception V3 & 84 & - & - & - & - & - \\
\hline Islam and Matin 2020 (DA, PPI) & Modelo Próprio & 86,0 & - & - & 85,0 & 87,0 & - \\
\hline Carvalho et al. 2020 & LeNet5 + XGBoost & 95,0 & - & - & 94,9 & 95,0 & 95,0 \\
\hline Yener and Oktay 2020 (TA, PPI) & VGG16 & 93,0 & - & - & 91,0 & 94,0 & 93,0 \\
\hline Serte and Serener 2020 (PPI, TA) & ResNet50 & 95,0 & 98,0 & 84,0 & - & - & 76,0 \\
\hline Kamel et al. 2021 (PPI, TA) & VGG19 & 98,3 & - & - & 95,6 & 97,3 & - \\
\hline Ardakani et al. 2020 (PPI, TA) & ResNet-101 & 99,5 & 100 & 99,0 & - & - & 99,4 \\
\hline Gifani et al. 2021 (PPI, TA) & Modelo Próprio & 85,2 & - & - & 85,7 & 85,2 & 91,0 \\
\hline Guranj et al. 2020 (TA) & Modelo Próprio & 99,1 & 97,3 & 99,9 & - & - & - \\
\hline Rahimzadeh et al. 2021 (PPI) & Modelo Próprio & 98,4 & 94,9 & 94,7 & 81,2 & - & - \\
\hline Hernández Santa Cruz 2021 (PPI, DA, TA) & Modelo Próprio & 86,7 & - & 89,5 & 88,1 & 85,8 & 90,8 \\
\hline Mishra et al. 2021 (DA, TA) & Modelo Próprio & 99,0 & - & - & - & - & - \\
\hline Jia et al. 2021 & Modelo Próprio & 99,3 & 99,2 & - & - & - & - \\
\hline Turkoglu 2021 (PPI, DA, TA) & Modelo Próprio + DenseNet201 & 98,3 & - & - & - & - & - \\
\hline Hu et al 2021 (PPI) & Modelo Próprio & 99,4 & 99,3 & - & 99,5 & 99,4 & 99,9 \\
\hline Di et al. 2021 (PPI) & Modelo Próprio & 89,7 & 93,2 & 84,0 & - & - & - \\
\hline Zhou et al. 2021 (TA, PPI) & Modelo Próprio & 99,0 & 99,0 & 99,6 & - & 98,5 & - \\
\hline Li et al. 2021 (PPI, TA) & CheXNet & 87,0 & - & - & - & 86,0 & 75,0 \\
\hline Arora et al. 2021 (PPI, DA, TA) & MobileNet & 100 & - & - & 100 & 100 & - \\
\hline
\end{tabular}

em conjuntos de dados com bastante amostras e classes diferentes, além de testar novos métodos de pré-processamento de imagens. Pretendemos também fazer uma otimização dos hiper-parâmetros nas melhores redes pesquisadas.

\section{Referências}

Abdar, A. K., Sadjadi, S. M., Soltanian-Zadeh, H., Bashirgonbadi, A., and Naghibi, M. (2020). Automatic detection of coronavirus (covid-19) from chest ct images using vgg16-based deep-learning. In 2020 5th ICBME, pages 212-216.

Ardakani, A., Kanafi, A., Acharya, U., Khadem, N., and Mohammadi, A. (2020). Application of deep learning technique to manage covid-19 in routine clinical practice using ct images: Results of 10 convolutional neural networks. Computers in Biology and Medicine, 121:103795.

Arora, V., Ng, E., Leekha, R., Darshan, M., and Singh, A. (2021). Transfer learningbased approach for detecting covid-19 ailment in lung ct scan. Computers in Biology and Medicine, 135:104575.

Berrimi, M., Hamdi, S., Cherif, R. Y., Moussaoui, A., Oussalah, M., and Chabane, M. (2021). Covid-19 detection from xray and ct scans using transfer learning. In International Conference of Women in Data Science at Taif University, pages 1-6.

Cai, X., Wang, Y., Sun, X., Liu, W., Tang, Y., and Li, W. (2020). Comparing the performance of resnets on covid-19 diagnosis using ct scans. In 2020 CITS, pages 1-4.

Carvalho, E., Carvalho, E., Carvalho Filho, A., de Araújo, F., and Rabêlo, R. (2020). Diagnosis of covid-19 in ct image using cnn and xgboost. In 2020 ISCC, pages 1-6. 
Castiglione, A., Vijayakumar, P., Nappi, M., Sadiq, S., and Umer, M. (2021). Covid-19: Automatic detection of the novel coronavirus disease from ct images using an optimized convolutional neural network. IEEE Trans. Ind. Informat., 17(9):6480-6488.

Cruz, J. (2021). An ensemble approach for multi-stage transfer learning models for covid19 detection from chest ct scans. volume 5, page 100027. Intelligence-Based Medicine.

Dan-Sebastian, B., Delia-Alexandrina, M., Sergiu, N., and Radu, B. (2020). Adversarial graph learning and deep learning techniques for improving diagnosis within ct and ultrasound images. In 2020 IEEE 16th ICCP, pages 449-456.

Di, D., Shi, F., Yan, F., Xia, L., Mo, Z., Ding, Z., Shan, F., Song, B., Li, S., Wei, Y., Shao, Y., Han, M., Gao, Y., Sui, H., Gao, Y., and Shen, D. (2021). Hypergraph learning for identification of covid-19 with ct imaging. Medical Image Analysis, 68:101910.

Dutta, P., Roy, T., and Anjum, N. (2021). Covid-19 detection using transfer learning with convolutional neural network. In 2021 2nd ICREST, pages 429-432.

Farias, L., Strabelli, D., Fonseca, E., Loureiro, B., Nomura, C., and Sawamura, M. (2020). Alterações tomográficas torácicas em pacientes sintomáticos respiratórios com a covid19. volume 53. Radiologia Brasileira.

Gifani, P., Shalbaf, A., and Vafaeezadeh, M. (2021). Automated detection of covid-19 using ensemble of transfer learning with deep convolutional. International Journal of Computer Assisted Radiology and Surgery, 16.

Gunraj, H., Wang, L., and Wong, A. (2020). Covidnet-ct: A tailored deep convolutional neural network design for detection of covid-19 cases from chest ct images. Frontiers in Medicine, 7:1025.

Hu, K., Huang, Y., Huang, W., Tan, H., Chen, Z., Zhong, Z., Li, X., Zhang, Y., and Gao, $X$. (2021). Deep supervised learning using self-adaptive auxiliary loss for covid-19 diagnosis from imbalanced ct images. volume 458, pages 232-245.

Islam, M. R. and Matin, A. (2020). Detection of covid 19 from ct image by the novel lenet-5 cnn architecture. In $202023 \mathrm{rd}$ ICCIT, pages 1-5.

Jia, G., Lam, H.-K., and Xu, Y. (2021). Classification of covid-19 chest X-ray and ct images using a type of dynamic cnn modification method. Computers in Biology and Medicine, 134:104425.

Júnior, E. A. and Yamashita, H. (Maio 2001). Aspectos básicos de tomografia computadorizada e ressonância magnética. Brazilian Journal of Psychiatry, 23.

Kamel, M. A., Abdelshafy, M., AbdulRazek, M., Abouelkhir, O., Fawzy, A., and Sahlol, A. T. (2021). Efficient classification approach based on covid-19 ct images analysis with deep features. In 2021 ICCCIS, pages 459-464.

Kitchenham, B. (2004). Procedures for performing systematic reviews. Keele, UK, Keele University, 33(2004):1-26.

Lai, Yaoming e Li, G., Wu, D., Lian, W., Li, C., Tian, J., Ma, X., Chen, H., Xu, W., Wei, J., Zhang, Y., and Jiang, G. (2020). 2019 novel coronavirus-infected pneumonia on ct: A feasibility study of few-shot learning for computerized diagnosis of emergency diseases. IEEE, 8:194158-194165. 
Li, C., Yang, Y., Liang, H., and Wu, B. (2021). Transfer learning for establishment of recognition of covid-19 on ct imaging using small-sized training datasets. KnowledgeBased Systems, 218:106849.

Mahase, E. (2020). Coronavirus: covid-19 has killed more people than sars and mers combined, despite lower case fatality rate. volume 368. BMJ.

Mishra, N., Singh, P., and Joshi, S. (2021). Automated detection of covid-19 from ct scan using convolutional neural network. Biocybernetics and Biomedical Engineering, $41: 572-588$.

Organization, W. H. (2020). Coronavirus. World Health Organization.

Ponti, M. A. and da Costa, G. B. P. (Tópicos em Gerenciamento de Dados e Informacões, 2017). Como funciona o deep learning. SBC, la ed., 23.

Rahimzadeh, M., Attar, A., and Sakhaei, S. M. (2021). A fully automated deep learningbased network for detecting covid-19 from a new and large lung ct scan dataset. In Biomedical Signal Processing and Control, page 102588.

Sari, I. P., Widodo, Nugraheni, M., and Wanda, P. (2020). A basic concept of image classification for covid-19 patients using chest ct scan and convolutional neural network. In 2020 1st ICITAMEE, pages 175-178.

Serte, S. and Serener, A. (2020). Discerning covid-19 from mycoplasma and viral pneumonia on ct images via deep learning. In 2020 4th ISMSIT, pages 1-5.

Seum, A., Raj, A. H., Sakib, S., and Hossain, T. (2020). A comparative study of cnn transfer learning classification algorithms with segmentation for covid-19 detection from ct scan images. In 2020 11th ICECE, pages 234-237.

Turkoglu, M. (2021). Covid-19 detection system using chest ct images and multiple kernels-extreme learning machine based on deep neural network. IRBM, 42:207-214.

Wang, W., Xu, Y., Gao, R., Lu, R., Han, K., Wu, G., and Tan, W. (2020a). Detection of SARS-CoV-2 in Different Types of Clinical Specimens. pages 1843-1844. JAMA.

Wang, X., Deng, X., Fu, Q., Zhou, Q., Feng, J., Ma, H., Liu, W., and Zheng, C. (2020b). A weakly supervised framework for covid-19 classification and lesion localization from chest ct. IEEE Transactions on Medical Imaging, 39(8):2615-2625.

Wang, Z., Liu, Q., and Dou, Q. (2020c). Contrastive cross-site learning with redesigned net for covid-19 ct classification. IEEE J. Biomed. Health Inform., 24(10):2806-2813.

Yener, F. M. and Oktay, A. B. (2020). Diagnosis of covid-19 with a deep learning approach on chest ct slices. In 2020 TIPTEKNO, pages 1-4.

Zhang, Y.-D., Satapathy, S. C., Zhu, L.-Y., Górriz, J. M., and Wang, S.-H. (2020). A seven-layer convolutional neural network for chest ct based covid-19 diagnosis using stochastic pooling. pages 1-1. IEEE Sensors Journal.

Zhou, T., Lu, H., Yang, Z., Qiu, S., Huo, B., and Dong, Y. (2021). The ensemble deep learning model for novel covid-19 on ct images. Applied Soft Computing, 98:106885. 